\title{
COVID 19 infection associated with thrombotic thrombocytopenic purpura
}

\author{
Gaurav Dhingra $^{1} \cdot$ Manideepa Maji $^{2} \cdot$ Saikat Mandal $^{3}$ (D) Sudeep Vaniyath ${ }^{1} \cdot$ Gita Negi $^{4} \cdot$ Uttam Kumar Nath $^{1}$
}

Accepted: 13 April 2021 / Published online: 27 April 2021

( ) The Author(s), under exclusive licence to Springer Science+Business Media, LLC, part of Springer Nature 2021

\begin{abstract}
Thrombotic thrombocytopenic purpura (TTP) which can cause significant mortality is a thrombotic microangiopathy due to deficiency of VWF cleaving protease ADAMTS13 and as per medical literature there are examples that TTP can be caused by COVID 19 infection. A 35 years old female after admission with right sided weakness and slurring of speech was found to be COVID positive and diagnosed as a case of TTP. Patient had absent ADAMTS13 level on day 1. Treatment was started with therapeutic plasma exchange (TPE) later injection Vincristine and Rituximab was given after 4th TPE as it was suspected as refractory case. Finally patient received 16 TPE procedures with cryo poor plasma as exchange fluid and gradually her platelet count started to maintain normal and she was discharged. Specific management and such association of this type of cases need to be studied more judiciously.
\end{abstract}

Keywords Thrombotic thrombocytopenic purpura $\cdot$ Covid $19 \cdot$ Thrombosis $\cdot$ Therapeutic plasma exchange $\cdot$ Vincristine in TTP

Highlights

- We hypothesize that in some patients similar to influenza, HIV and HTLV, COVID-19, a single stranded RNA virus may also provoke predominantly IgG type autoantibody production which may interact against ADAMTS13.

- As a result, VWF-ADAMTS 13 axis get more dysregulated and this in turn causes the accumulation of ultralarge VWF multimers, which, in presence of high shear stress, spontaneously aggregate platelets leading to uncontrolled thrombus formation in the microcirculation and eventually patient presents with TTP.

- In this index case also we found presence of high titer inhibitor (3 Bethesda unit). This finding supports our hypothesis.

Saikat Mandal

saikatmandal500@gmail.com

1 Medical Oncology Hematology, AIIMS Rishikesh, Rishikesh, India

2 Pediatric Hematology Oncology, Superspeciality Pediatric Hospital \& PG Teaching Institute, Noida, India

3 Transfusion Medicine, Sarvodaya Hospital \& Research Centre, Delhi NCR, India

4 Transfusion Medicine, AIIMS Rishikesh, Rishikesh, India
At the end of December 2019 severe acute respiratory syndrome coronavirus 2 (SARS-CoV-2/COVID-19) related cases emerged and in February 2020, WHO declared it as a Pandemic [1]. As per medical literature it is evident that COVID 19 cases may show multiple immunological phenomenon like Immune thrombocytopenic purpura, GuillainBarré syndrome and antiphospholipid syndrome, autoimmune haemolytic anaemia, Thrombotic thrombocytopenic purpura (TTP) [2-6]. TTP is a thrombotic microangiopathy, mainly defined by the pentad of fever, renal dysfunction, hemolytic anemia, thrombocytopenia, central nervous system (CNS) involvement due to deficiency of VWF cleaving protease ADAMTS13. TTP require to be accurately diagnosed and promptly intervened otherwise patient can have mortality rate up to $90 \%$.

We report a case of 35 years old female patient without any comorbidity and significant past medical history or family history of genetic or autoimmune disease presented to our hospital with acute onset right sided weakness, slurring of speech, seizure and bi-cytopenia with preceding history of loose stools 15 days back. After admission, her nasopharyngeal and oropharyngeal swab for COVID-19 was found to be positive by RT-PCR method. MRI brain showed small infarct on left parietal lobe.

On admission the patient was alert, conscious, oriented, afebrile with stable vitals. Laboratory investigations showed 
anemia $(\mathrm{Hb}-8.25 \mathrm{gm} / \mathrm{dL})$ with thrombocytopenia (Platelet-20,000/ $\mu \mathrm{L}$ ) with signs of hemolysis and $8 \%$ schistocytes on peripheral smear (Fig. 1). All these findings are clinically suggestive of TTP. To rule out secondary cause of TTP and other causes anemia with thrombocytopenia, patient was evaluated for antinuclear antibody (ANA), anticardiolipin antibody (IgM and $\operatorname{IgG}$ ), beta 2 glycoprotein (IgG and $\operatorname{IgM}$ ) and paroxysmal nocturnal hemoglobinuria $(\mathrm{PNH})$, which were all negative. Bone marrow aspiration and biopsy was also done which was normal. Indirect antiglobulin test and direct antiglobulin test which were done to rule out Evans Syndrome were found to be negative. So, she was started with injection Methyl prednisolone (1gm) and therapeutic plasma exchange (TPE) (1.5 volume exchange with cryo poor plasma (CPP) as replacement fluid) was initiated. Therapeutic plasma exchange was performed daily with regular monitoring of complete blood count, lactate dehydrogenase and reticulocyte count. Patient started responding well to the treatment. [Summary of day wise investigations are shown in Table 1.] To confirm the diagnosis of TTP, ADAMTS 13 level were sent on day 1 which was reported as absent levels with presence of high titer inhibitor (3 Bethesda unit). On day 6 , her platelets count improved till $1,28,000 / \mu \mathrm{L}$ but then gradually decreased to $16,650 / \mu \mathrm{L}$ with one episode of vaginal bleed on day 12 . As the patient was hepatitis B core antibody positive and viral load report awaited so, Inj. VINCRISTINE $\left(1.4 \mathrm{mg} / \mathrm{m}^{2}\right)$ and single donor platelet transfusion were given on day 12 of illness and daily 1.5 volume plasma exchange was continued with cryo poor plasma as replacement fluid. From day 14, onwards platelet counts showed increasing trends. As HBV DNA reports were negative so patient was started on Inj Rituximab $\left(375 \mathrm{mg} / \mathrm{m}^{2}\right)$ with prophylactic tab Entecavir. On day 17, platelet count became more than $1,50,000 / \mu \mathrm{L}$ with normal serum $\mathrm{LDH}$ level. So patient was given two cycles of maintenance TPE with 1 volume CPP as replacement fluid. As patient continued to have improving laboratory parameters so she was discharged on day 23. So, patient received total 16 cycles of TPE and two doses of Inj. Vincristine $\left(1.4 \mathrm{mg} / \mathrm{m}^{2}\right)$ and one dose of Inj Rituximab $\left(375 \mathrm{mg} / \mathrm{m}^{2}\right)$ before discharge. After discharge patient received 3 more cycles of weekly Inj Rituximab $\left(375 \mathrm{mg} / \mathrm{m}^{2}\right)$. She has been regularly followed since last 6 months with complete blood count, LFT, serum LDH. She is completely asymptomatic with normal laboratory parameters.

As per medical literature viruses may play an important role in the pathogenesis of TTP. The exact mechanism of thrombotic microangiopathy may vary with different viral diseases. DNA viruses like CMV, HHV 8 had been thought to cause TTP by direct endothelial injury and cytokine storm whereas RNA viruses like HIV, HTLV, influenza had been implicated to cause TTP by producing ADAMTS13 inhibitor and direct endothelial injury [7]. According to case reports

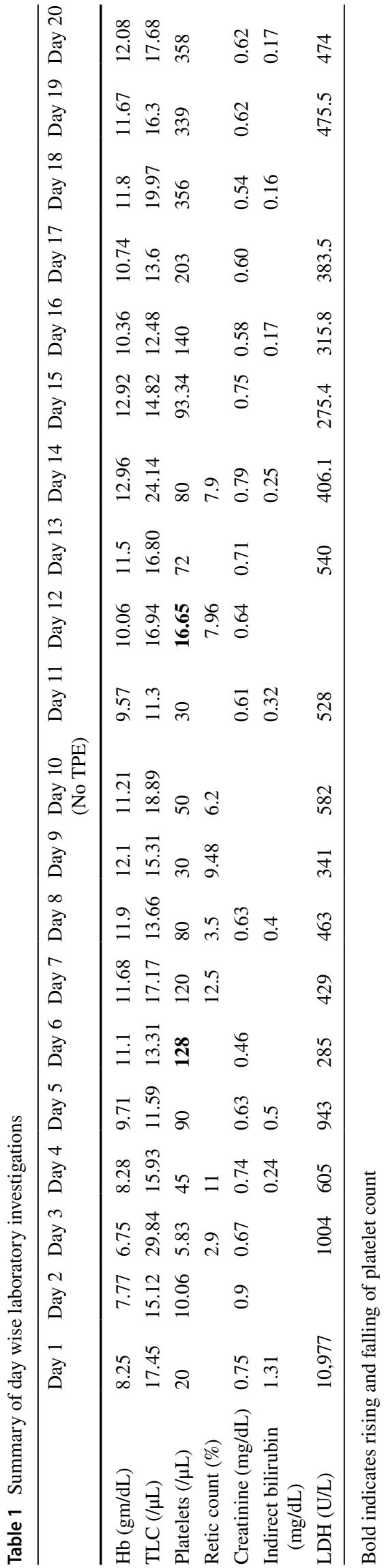


by Kosugi et al. and Joseph et al., ADAMTS13 activity was depleted in 2 patients diagnosed with RNA viruses Influenza A; both demonstrated increased anti-ADAMTS13 antibody concentrations $[8,9]$. While Capecchi et al. hypothesized that by causing a severe systemic inflammation in COVID 19 led to diffuse activation of the microvascular endothelium that triggered the acute TTP [10]. There are already multiple case reports on the association of COVID 19 and TTP [11, 12].

Study done by Mancini et al., Bazzan et al. and Huisman and Sikma showed that there is a state of hypercoagulability in COVID 19 patients driven by high factor VIII levels, and a perturbation of the endothelium, with elevated VWF and complement activation and there was a mild to moderate reduction of ADAMTS13 activity [13-15]. So, it is well understood that there is already a state of shifted equilibrium between VWF, endothelium, platelet and ADAMTS13 towards spontaneous activation. We hypothesize that in some patients similar to influenza, HIV and HTLV, COVID19 , a single stranded RNA virus may also provoke predominantly IgG type autoantibody production which may interact against ADAMTS13. As a result, VWF-ADAMTS 13 axis get more dysregulated and this in turn causes the accumulation of ultra-large VWF multimers, which, in presence of high shear stress, spontaneously aggregate platelets leading to uncontrolled thrombus formation in the microcirculation and eventually patient presents with TTP. In this index case also we found presence of high titer inhibitor (3 Bethesda unit). This finding supports our hypothesis.

American Society of Haematology suggests about judicious use of Rituximab in TTP as it has been associated with viral reactivation and potentially an increased risk of primary viral infections, given impact on antibody production and they recommends Rituximab administration could be delayed until the acute COVID-19 infection has cleared and neutralizing COVID- 19 antibodies have already been produced [16]. So, in this case Rituximab therapy was started delayed as anti-HBc was found to be positive although the

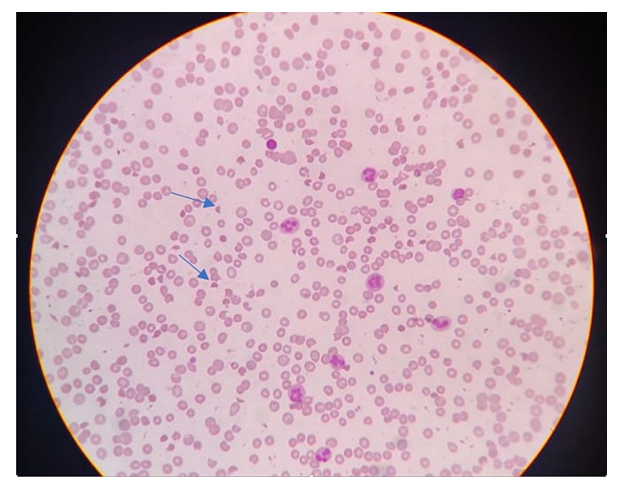

Fig. 1 Under $\times 40$ microscopy peripheral blood smear of the patient shows numerous schistocytes (indicated by blue arrows) treatment must be started with methyl prednisolone and TPE based on clinical presentation without waiting for conclusive evidence in favour of diagnosis of TTP. As the patient suddenly deteriorated after initial improvement two doses of Vincristine was given keeping the refractory TTP as a possibility and uneventful course of COVID related patients treated with Vincristine [17, 18].

Though the pathophysiology is not well established after going through recent medical literature review it is obvious that there is a possibility of such an association between COVID and TTP. Specific management and such association of this type of cases need to be studied more judiciously.

\section{Declarations}

Conflict of interest The authors declare that they have no conflict of interest.

\section{References}

1. World Health Organization (2020) Director-general's remarks at the media briefing on 2019-nCoV. https://www.who.int/dg/speec hes/detail/who-director-general-s-remarks-at-the-media-briefingon-2019-ncov-on-11-february-2020. Accessed 11 Nov 2020.

2. Zhang Y, Xiao M, Zhang S, Xia P, Cao W, Jiang W, Chen H, Ding X, Zhao H, Zhang H et al (2020) Coagulopathy and antiphospholipid antibodies in patients with Covid-19. N Engl J Med 382:e38

3. Zulfiqar AA, Lorenzo-Villalba N, Hassler P, Andrès E (2020) Immune thrombocytopenic purpura in a patient with Covid-19. N Engl J Med 382(18):e43

4. Bomhof G, Mutsaers PGNJ, Leebeek FWG, Te Boekhorst PAW, Hofland J, Croles FN, Jansen AJG (2020) COVID-19-associated immune thrombocytopenia. Br J Haematol. https://doi.org/10. 1111/bjh. 16850

5. Lopez C, Kim J, Pandey A, Huang T, DeLoughery TG (2020) Simultaneous onset of COVID-19 and autoimmune haemolytic anaemia. Br J Haematol. https://doi.org/10.1111/bjh.16786

6. Hindilerden F, Yonal-Hindilerden I, Akar E, Kart-Yasar K (2020) Covid-19 associated autoimmune thrombotic thrombocytopenic purpura: report of a case. Thromb Res 1(195):136-138

7. da Silva RL (2011) Viral-associated thrombotic microangiopathies. Hematol Oncol Stem Cell Ther 4(2):51-59

8. Kosugi N, Tsurutani Y, Isonishi A, Hori Y, Matsumoto M, Fujimura Y (2010) Influenza A infection triggers thrombotic thrombocytopenic purpura by producing the anti-ADAMTS13 IgG inhibitor. Intern Med 49:689-693. https://doi.org/10.2169/ internalmedicine.49.2957

9. Joseph A, Fangio P, Barbier C, Hayon J, Loubières Y, Pichereau C, Outin H, Alves M (2016) Seasonal flu as a triggering factor for acquired thrombotic thrombocytopenic purpura. J Hematol Thromboembolic Dis. https://doi.org/10.4172/2329-8790.10002 43

10. Capecchi M, Mocellin C, Abbruzzese C, Mancini I, Prati D, Peyvandi F (2020) Dramatic presentation of acquired TTP associated 
with COVID-19. Haematologica 105(10):262345. https://doi.org/ 10.3324/haematol.2020.262345

11. Hindilerden F, Yonal-Hindilerden I, Akar E, Kart-Yasar K (2020) Covid-19 associated autoimmune thrombotic thrombocytopenic purpura: report of a case. Thromb Res 195:136-138

12. Albiol N, Awol R, Martino R (2020) Autoimmune thrombotic thrombocytopenic purpura (TTP) associated with COVID-19. Ann Hematol 28:1

13. Mancini I, Baronciani L, Artoni A et al (2021) The ADAMTS13von Willebrand factor axis in COVID-19 patients. J Thromb Haemost 19:513-521. https://doi.org/10.1111/jth.15191

14. Bazzan M, Montaruli B, Sciascia S, Cosseddu D, Norbiato C, Roccatello D (2020) Low ADAMTS 13 plasma levels are predictors of mor- tality in COVID-19 patients. Intern Emerg Med 15(5):861-863. https://doi.org/10.1007/s11739-020-02394-0

15. Huisman A, Beun R, Sikma M, Westerink J, Kusadasi N (2020) Involvement of ADAMTS13 and von Willebrand factor in thromboembolic events in patients infected with SARS-CoV-2. Int J Lab Hematol 42(5):e211-e212. https://doi.org/10.1111/ijlh.13244
16. COVID-19 and TTP-Hematology.org. https://www.hematology. org:443/covid-19/covid-19-and-ttp. Accessed 30 Oct 2020

17. Ziman A, Mitri M, Klapper E, Pepkowitz SH, Goldfinger D (2005) Combination vincristine and plasma exchange as initial therapy in patients with thrombotic thrombocytopenic purpura: one institution's experience and review of the literature. Transfusion 45(1):41-49. https://doi.org/10.1111/j.1537-2995.2005.03146.x

18. Cheung CKM, Law MF, Lui GCY, Wong SH, Wong RSM (2019) Coronavirus disease 2019 (COVID-19): a haematologist's perspective. Acta Haematol 2020:1-14. https://doi.org/10.1159/ 000510178

Publisher's Note Springer Nature remains neutral with regard to jurisdictional claims in published maps and institutional affiliations. 